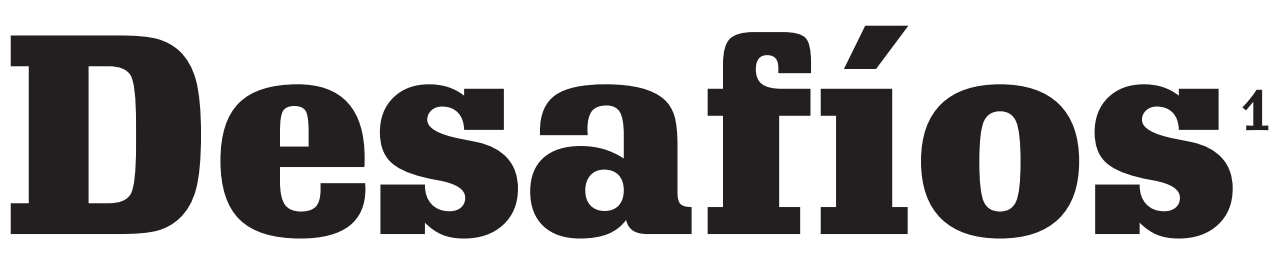

\title{
para la pedagogía en los tiempos actuales
}

Francisco Sorto Rivas

\section{Síntesis}

Este artículo versa sobre los desafíos que representa para la "escuela", como institución moderna fundamental para la socialización del individuo y la preparación para su integración al sistema de producción imperante, como resultado de la descomposición del núcleo familiar — base de la sociedad_-, la cual está impactando negativamente sobre la formación e inducción del individuo, en el hogar.

\section{Palabras claves:}

Aprendizaje, Educación, Escuela, Individuo, Socialización.

\section{Introducción}

$\mathbf{G}$ omo consecuencia natural del modo de producción capitalista, las condiciones económicas han requerido, gradualmente, que ambos padres de familia se incorporen al mercado laboral o deban desplazarse, inclusive, fuera del país (emigrar), con lo cual se ha sacrificado parte de las funciones formativas de sus hijos, las cuales han quedado a cargo de personas ajenas a la familia, de quienes reciben esta inducción en edades tempranas, por decirlo de alguna manera, por lo que, la labor de socialización adicional o complementaria que debería desempeñar la escuela se vuelve crítica, porque muchas veces viene a suplir, completamente, esta tarea formativa del joven destinada a garantizar su adaptación a la sociedad y su aceptación de las instituciones que la rigen.

\section{Contenido}

A lo largo de la historia de la pedagogía se han discutido, con diferentes matices, tres problemas fundamentales que tienen que ver con el ámbito de la educación, los métodos de enseñanza y el propósito mismo de la educación en la sociedad. En ese sentido, los paradigmas que han guiado el análisis de estos problemas han dado forma a esta ciencia en el tiempo, sirviéndose de ella incluso para obstaculizar, promover o consolidar proyectos de ingeniería social, pudiendo destacarse algunos hitos como el uso de la pedagogía para instaurar regímenes como los denominados "democrático-liberales" que, llevados a una situación límite como modelo sociológico, pueden derivar en regímenes políticos autoritarios como sucedió con el nacional-socialismo

1/ Este artículo está inspirado en el libro del Hno. Eugenio Magdaleno, titulado La Educación, llave del Tercer Milenio, de la colección respuestas educativas, editorial Magisterio del Río de la Plata, Argentina 1999

16 


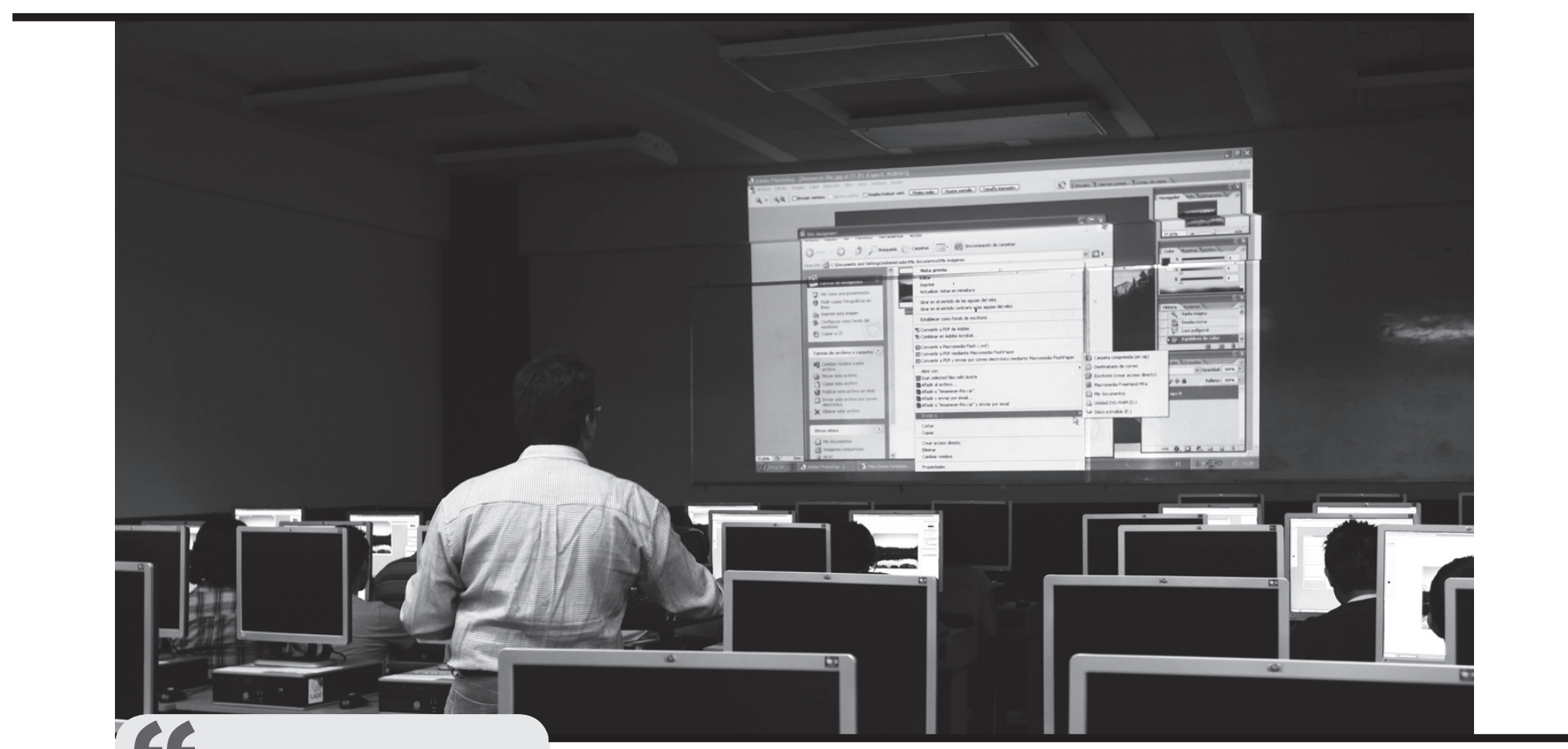

La escuela como

\section{institución moderna}

debe gestionar así

esta nueva realidad

cambiante, revisar

su oferta educativa,

sus proyectos

extracurriculares $\mathbf{y}$

los medios didácticos

disponibles para los

docentes, a fin de atender

de manera integral

las necesidades de los

estudiantes en las aulas

y fuera de ellas. que se apoyó en la pedagogía, desde su vertiente conductivista, para promover y justificar la existencia de una sociedad intolerante frente a otras culturas. Además, tenemos la experiencia reciente de la pedagogía soviética, que marcó cierta transición entre el conductivismo y el constructivismo -al menos desde el punto de vista formal- y que estuvo precedida por una preocupación más "emancipadora".2

No obstante que los tres aspectos arriba señalados apuntan a problemas vigentes todavía para la ciencia de la educación, el contexto histórico actual introduce nuevos elementos para el análisis, particularmente porque la pedagogía juega un papel irremplazable en el proceso de socialización del individuo, especialmente por la etapa temprana en que se incorpora a la escuela, entendida esta como aquella institución moderna, fruto del ingenio humano, a través de la cual se implanta la práctica educativa correspondiente.

Dado que el proceso de enseñanza trasciende el interés de la sociedad por el desarrollo de habilidades cognitivas e instrumentales del ser humano y busca cultivar en él actitudes integrativas para la convivencia y el progreso social, las condiciones del entorno actual en que se mueven los individuos exigen una reflexión fresca sobre otros problemas que demandan respuestas pedagógicas, aprovechando las experiencias acumuladas, hasta la fecha, sobre cómo aprende el ser humano.

\footnotetext{
2 / El enfoque conductivista de la teoría curricular tiene como paradigma la práctica instrumental de la educación, en función de la necesidades históricas de la sociedad, relegando a un segundo plano la realización del educando como persona; mientras que el constructivismo considera, además, el contexto social en que se desenvuelve y las necesidad no materiales de la persona.
} 
96 No hay que olvidar que la pedagogía es la ciencia que estudia cómo aprendemos los humanos en un medio social; los esquemas convencionales de enseñanza resultan ineficaces hoy. $y$

Ese sentido de urgencia proviene del hecho que la concepción convencional de la escuela se encuentra en el centro de un debate permanente, dado que, para una parte significativa del colectivo imaginario, esta ha dejado de representar un complemento del proceso de aprendizaje iniciado en el hogar, para convertirse, prácticamente, en un sustituto total de la labor formativa realizada por los padres en la casa, ya que la ausencia prolongada de estos tiende a profundizarse debido a las exigencias laborales del mundo moderno. Esta situación se observa claramente en nuestro ambiente local, donde tanto la madre como el padre trabajan jornadas extendidas, delegando inconscientemente con ello las funciones formativas, afectivas y de socialización de los hijos en personas ajenas al núcleo familiar.

No obstante lo anterior, la principal fuente de problematización que demanda replantear el rumbo de la pedagogía y de la escuela como institución moderna proviene de la desintegración familiar por las corrientes migratorias de grupos importantes de nuestra población, por factores económicos y por la transculturación provocada, también, por la separación de familias enteras, que, en conjunto, explican la falta de modelos de autoridad paterna y materna en algunos hogares, tan fundamentales para el desarrollo integral del niño y de la juventud en general. Estos vacíos conductuales están siendo llenados por otras formas de organización alternativas, como son las "maras", a manera de caso extremo y connotación negativa.

Estas condiciones de fragmentación y desintegración de las familias están generando altos niveles de violencia o agresividad, al menos, en muchos centros de estudio, donde la agresión no se limita a los compañeros de clases o de estudio, sino que se extiende al mismo cuerpo docente, que son una forma de expresión visible de las estructuras de autoridad y poder objetivado de la sociedad ante los estudiantes, las cuales no son toleradas - siquiera- por muchos de ellos que se ven agobiados por la desorientación, la desesperanza acerca de su futuro, y experimentan, además, diversos grados de privación social.

Este ambiente de trabajo ha contribuido al deterioro en la calidad educativa, porque los docentes se sienten amenazados por sus propios alumnos, quienes retan constantemente la autoridad del maestro en las aulas; igualmente sucede con los estudiantes que desearían estudiar en un ambiente más aséptico y prefieren dejar de asistir a clases por temor a ser victimizados por sus compañeros.

Esta problemática exige de la pedagogía un mayor esfuerzo de análisis para responder a este fenómeno que atenta contra todas las instituciones surgidas de la primera modernidad en general, ${ }^{3}$ incluyendo obviamente la institución denominada escuela.

Es importante señalar aquí, que esta realidad no debería representar algún desaliento para la pedagogía como ciencia, sino que un acicate para tratar de comprenderla en su dimensión correcta, y adaptarse mejor a las nuevas exigencias, ya que, regresando a los problemas históricos de la pedagogía, como son los contenidos, los métodos de estudio y los fines de la educación, esta parece carecer de sentido para muchos estudiantes en la actualidad.

Eso lo podemos observar en el sistema educativo nacional, que debería servirnos de laboratorio para examinar qué está pasando con la organización social posmoderna, donde los niveles de violencia son crecientes; el desinterés por aprender, notorio, y la desorientación respecto a la vocación por profesionalizarse, palpable. Además, muchas veces la visión reduccionista que se tiene a la hora de formular políticas públicas impide aprovechar todo su potencial en esta época de crisis de valores y descomposición de la familia nuclear. ${ }^{4}$

La escuela como institución moderna debe gestionar así esta

\footnotetext{
$\overline{3}$ / Se dice que la primera modernidad corresponde al período de creación de Estados modernos y soberanos; y segunda modernidad, al Estado actual de pérdida de poder por parte de este en diversos campos, debido al nivel de integración de las economías a escala global.

${ }^{4}$ / Si la escuela se ha convertido prácticamente en un sustituto circunstancial de la formación doméstica de nuestros hijos, sería necesario ampliar sus capacidades para llenar mejor las carencias afectivas que enfrentan muchos de ellos y despertar actitudes colaborativas hacia sus compañeros y maestros.
}

18 
nueva realidad cambiante, revisar su oferta educativa, sus proyectos extracurriculares y los medios didácticos disponibles para los docentes, a fin de atender de manera integral las necesidades de los estudiantes en las aulas y fuera de ellas. No hay que olvidar que la pedagogía es la ciencia que estudia cómo aprendemos los humanos en un medio social; los esquemas convencionales de enseñanza resultan ineficaces hoy, porque el entorno social de los educandos ha cambiado $y$, por ende, la forma en que aprenden.

De hecho, se han venido ampliando con el tiempo los espacios dejados por los padres -aunque sea por abandono fáctico, cuando los padres han tenido que emigrar, dejando atrás a sus hijos - en lo relativo a la educación inicial del niño en materia de socialización, de inducción para el sometimiento a las reglas de convivencia establecidas por la sociedad y para la subordinación a las instituciones creadas por el Estado para garantizar su funcionamiento, según lo señaló Émile Durkheim en su momento. A estos hechos sociales se refería este insigne pensador, bajo el título de anomias o desadaptación social; este fenómeno ha venido en crecimiento a lo largo de las últimas dos décadas, al menos, en $\mathrm{El}$ Salvador. ${ }^{5}$

La razón de recordar los aportes de Durkheim aquí obedece a que dicho fenómeno de descomposición corresponde a un hecho social ${ }^{6}$ y tiene que ver con la manera de actuar, pensar y sentir de una sociedad; en otras palabras, comprende tipos de conductas o pensamientos que están dotados de poder imperativo y coercitivo, en virtud de lo cual se imponen colectivamente a la gente.

A partir de lo anterior y dado que los hechos sociales no se crean, sino que se interiorizan por medio de la educación o socialización de la cual son objeto los individuos, las anomias observadas en las aulas, de violencia, desidia y desadaptación escolar, pueden resolverse desde el ámbito educativo, pero para ello se requiere de conciencia de parte de todos los responsables del sistema educativo, a fin de dotar a las escuelas de los medios necesarios para incidir positivamente sobre la actitud y la conducta de los jóvenes.

Esta labor de educación del individuo corre a cargo de diversas instituciones del Estado, par-ticularmente de la "escuela", especialmente ahora cuando unos de los protagonistas más importantes, como son los padres,

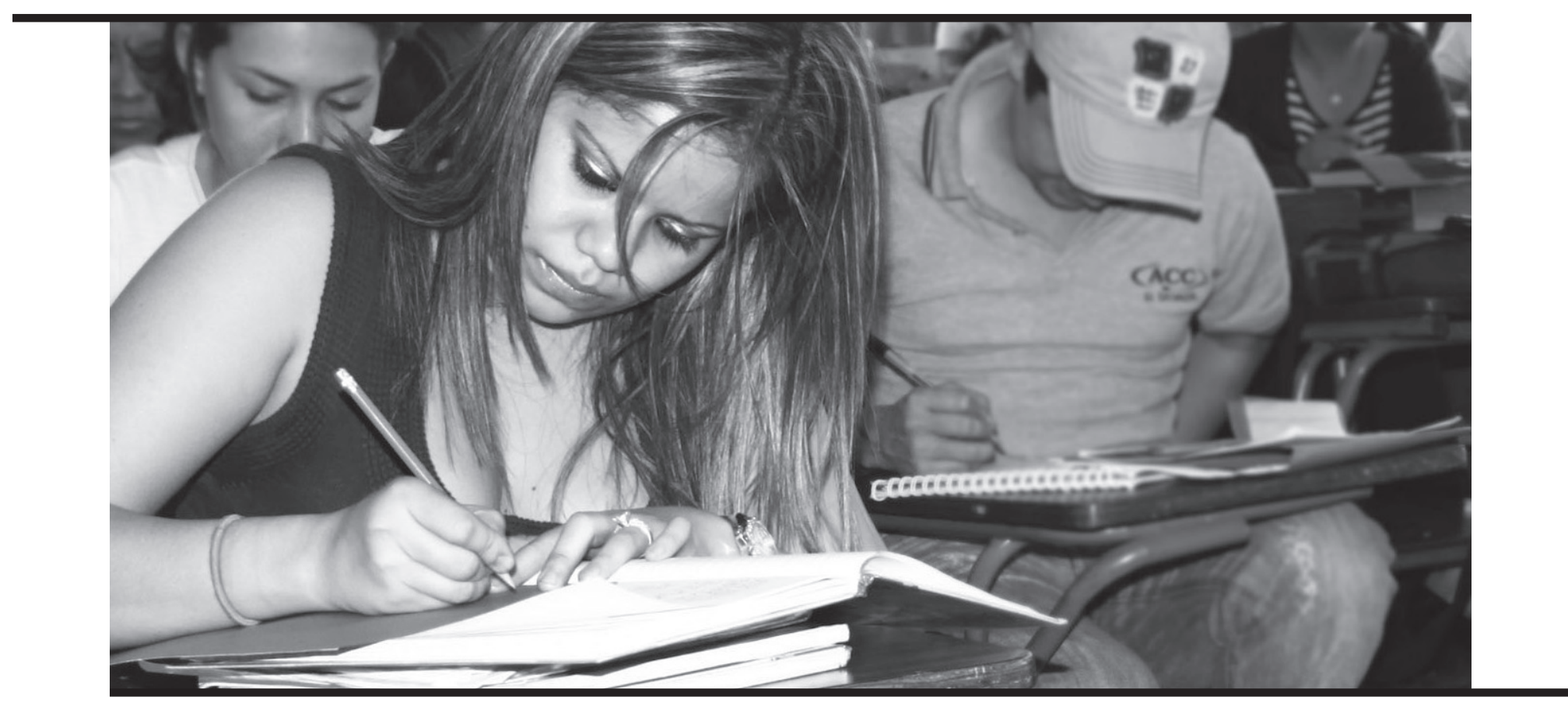
5 / Cuando Durkheim analizaba el funcionamiento de las sociedades complejas, (o modernas) señalaba que estas experimentan anomias, entendiendo por estas la carencia de solidaridad
social, debido a que las relaciones con los órganos no están reglamentadas debido al desajuste originado por la permanente modernización.
6 / ¿Qué es un hecho social? Es un fenómeno que tiene algún lugar en la sociedad. Y por ello es general y de interés social. Según Durkheim, un hecho social es toda forma de ejercer una
coacción exterior sobre el individuo; o bien, general para una sociedad dada y con existencia propia, independientemente de sus manifestaciones individuales.

Entorno ISSN: 2218-3345

Abril 2009, $\mathrm{N}^{\circ} .42$ 


\section{ent orno}
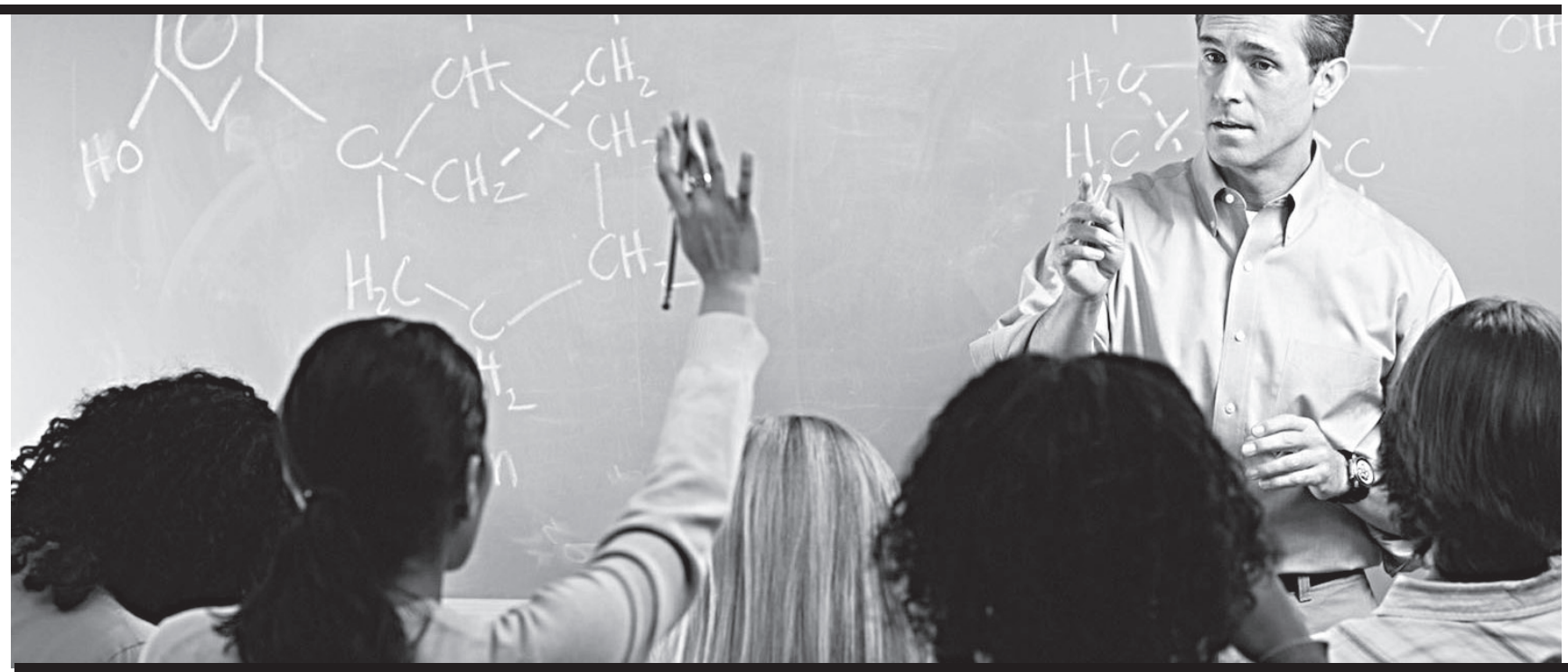

han ido perdiendo incidencia con el tiempo, por lo que esta debe llenar, de alguna manera, dicho vacío para evitar que la descomposición social y el abierto desafío de los jóvenes hacia las "instituciones modernas" continúe profundizándose.

La escuela está llamada, entonces, a convertirse en ese espacio de creación de valores y de respecto por las instituciones como la propiedad privada de los medios de producción, la religión y el Estado de derecho, a manera de ejemplos. Si los espacios de socialización a cargo del hogar han ido retrocediendo, no es extraño observar conductas antisociales en algunos de nuestros jóvenes, que ante la falta de orientación oportuna y consejos sinceros de parte de los padres, actúan impulsivamente y con algún grado de resentimiento hacia las instituciones que sustentan la organización social.

Estas pocas líneas deberían llamarnuestra atención sobre los hechos sociales que inciden sobre las actitudes y conductas de nuestra juventud (condiciones ambientales enque se están criando muchos de nuestros hijos) y el papel que debe jugar la educación en contravía a este entorno enrarecido y, más concretamente, la escuela como objetivación de dicha institución, como lo señalara claramente Max Weber al construir las bases de la sociología positivista; ${ }^{7}$ dicho papel debe descansar principalmente en los objetivos actitudinales de la didáctica (socializadores),másqueenlosconceptuales o procedimentales que son inculcados en el educando (individuo). Además, se pretende dejar sentada la inquietud que esta realidad debería permear las reformas educativas impulsadas en nuestra región. Es imperativo trascender la discusión de los contenidos, objetivos didácticos y métodos de enseñanza, hacia los desafíos impuestos por las nuevas condiciones ambientales en que se mueven los jóvenes.

La pedagogía debe examinar, con base en las condiciones imperantes, la mejor forma de suplir las carencias de los educandos, cómo adecuar los objetivos, contenidos, medios y materiales educativos a estas nuevas exigencias, las cuales deberán contemplar, indiscutiblemente, más trabajo extracurricular para llenar positivamente la falta que hace la función educadora de los padres, por un lado; mientras que por el otro, para absorber esa disponibilidad de tiempo libre con que cuentan los muchachos.

Se necesitan estrategias para convertir a la "escuela" en un espacio apropiado para suplir, aunque sea parcialmente, la ausencia de los padres dentro del proceso integral de socialización de los individuos; estando esta convocada para desempeñar un papel basal para transitar hacia otras formas de organización posmodernas, con relaciones sociales de producción distintas, aunque por ahora estas resulten todavía opacas para nuestra vista.

\section{Bibliografía:}

- escobar BAÑOS, Juan Carlos; Currículo: más allá de la teoría de la enseñanza, Editorial Abril Uno, EI Salvador 2007.

- MAGDALENO, Eugenio; La educación, llave del tercer milenio; Editorial Magisterio del Río de la Plata; Argentina 1999.

- NASSIF, Ricardo; Pedagogía general; Editorial Kapelusz, Argentina 1958.

7 / Max Weber tenía una visión más instrumental de las instituciones y las analizaba a partir de su sentido teleológico, es decir, de la finalidad que justificaba su existencia. 